\title{
The fungus Ustilago maydis and humans share disease-related proteins that are not found in Saccharomyces cerevisiae Martin Münsterkötter*1 and Gero Steinberg2,3
}

\author{
Address: ${ }^{1}$ Munich Information Center for Protein Sequences/Institute of Bioinformatics and Systems Biology, Helmholz Zentrum München, \\ Germany, ${ }^{2}$ Max-Planck-Institut für terrestrische Mikrobiologie, Marburg, Germany and ${ }^{3}$ School of Bioscience, Stocker Rd., Exeter University, EX4 \\ 4QD, UK \\ Email: Martin Münsterkötter* - M.Muensterkoetter@helmholtz-muenchen.de; Gero Steinberg - G.Steinberg@exeter.ac.uk \\ * Corresponding author
}

Published: 20 December 2007

BMC Genomics 2007, 8:473 doi:10.1/86/147|-2164-8-473
Received: 13 September 2007

Accepted: 20 December 2007

This article is available from: http://www.biomedcentral.com/I47I-2/64/8/473

(c) 2007 Münsterkötter and Steinberg; licensee BioMed Central Ltd.

This is an Open Access article distributed under the terms of the Creative Commons Attribution License (http://creativecommons.org/licenses/by/2.0), which permits unrestricted use, distribution, and reproduction in any medium, provided the original work is properly cited.

\begin{abstract}
Background: The corn smut fungus Ustilago maydis is a well-established model system for molecular phytopathology. In addition, it recently became evident that $U$. maydis and humans share proteins and cellular processes that are not found in the standard fungal model Saccharomyces cerevisiae. This prompted us to do a comparative analysis of the predicted proteome of $U$. maydis, S. cerevisiae and humans.

Results: At a cut off at $20 \%$ identity over protein length, all three organisms share 1738 proteins, whereas both fungi share only $54 \mathrm{I}$ conserved proteins. Despite the evolutionary distance between $U$. maydis and humans, 777 proteins were shared. When applying a more stringent criterion $(\geq 20 \%$ identity with a homologue in one organism over at least 50 amino acids and $\geq 10 \%$ less in the other organism), we found $68 \mathrm{I}$ proteins for the comparison of $U$. maydis and humans, whereas the both fungi share only 622 fungal specific proteins. Finally, we found that $S$. cerevisiae and humans shared 312 proteins. In the $U$. maydis to $H$. sapiens homology set 454 proteins are functionally classified and 42 proteins are related to serious human diseases. However, a large portion of 222 proteins are of unknown function.

Conclusion: The fungus $U$. maydis has a long history of being a model system for understanding DNA recombination and repair, as well as molecular plant pathology. The identification of functionally un-characterized genes that are conserved in humans and $U$. maydis opens the door for experimental work, which promises new insight in the cell biology of the mammalian cell.
\end{abstract}

\section{Background}

Fungi are a remarkably successful group of eukaryotes that play an essential part in our ecosystem as symbionts and decomposers of organic material $[1,2]$. On the other hand, numerous fungi are devastating human and plant pathogens that are a serious threat to agricultural industry and human health $[3,4]$. In addition, some fungi serve as simple eukaryotic model systems for basic cell biology questions, as they are closely related to animal cells $[5,6]$ and share important cellular processes. In this respect, the most prominent fungal model system is the budding yeast Saccharomyces cerevisiae. Its genomic sequence was among the first published in 1996 [7] and more than $77 \%$ of the 6100 genes are assigned to cellular functions [8]. However, this powerful model has its limitations, because certain basic processes found in animal cells, such as long- 
distance transport along the microtubule cytoskeleton or the removal of the nuclear envelope in mitosis do not exist in budding yeast $[9,10]$. In recent years, large scale sequencing projects were launched in order to obtain genome sequences from over 80 additional fungi [11]. Among the recently released genomes is that of the basidiomycete $U$. maydis [12], which is also known as a smut fungus on corn. Beside its pathogenic lifestyle and numerous technical advantages of this fungus, the recently published manually annotated proteome, which is available on the public server of the Munich Information Center for Protein Sequences (MIPS; $[13,14])$ established this fungus as a powerful model system for molecular phytopathology [15-17]. However, U. maydis also has a long standing history as a cell biological model system and important basic concepts, such as the molecular mechanism of DNA recombination (e.g. the Holiday Junction was initially described in this fungus $[18,19])$.

Recently, the importance of $U$. maydis as a model system increased, as studies on the microtubule cytoskeleton in polar growth and mitosis revealed that important processes are conserved between $U$. maydis and mammalians. Such processes are not found in the model fungus $S$. cerevisiae [20]. Among these are kinesin-1- and kinesin-3based transport processes [21-23], both of which motors are not found in the budding yeast. Another striking example is the removal of the nuclear envelope in mitosis. In contrast to budding yeast, the nuclear envelope is removed at the onset of mitosis in humans $[24,25]$ and in $U$. maydis [26]. Furthermore, in both organisms this is accompanied by the disassembly of the nuclear pores and the recruitment of some pore components to the mitotic chromosomes [27-29]. Interestingly, the mechanistically parallels are reflected by unexpected high sequence conservation of pore components [27]. This strongly suggests that sequence conservation between humans and $U$. maydis coincides with functional similarity. These data indicate that $U$. maydis and mammalian cells share common cellular processes and the underlying molecular machinery that are not found in S. cerevisiae. In order to investi- gate this further, we made use of the SIMAP (Similarity Matrix of Proteins) database, which is based on a SmithWaterman pair-wise comparison of all known predicted protein sequences available [30]. Using this bioinformatic resource we analyzed the manually annotated proteome set of $U$. maydis and $S$. cerevisiae and the currently accessible protein information of Homo sapiens. Surprisingly, we found that the proteome of $U$. maydis is more closely related to humans than to the fungal cousin S. cerevisiae. Using the FunCat database that summarizes predicted protein function [31], we demonstrate that many proteins conserved in $H$. sapiens and $U$. maydis can be assigned to certain cellular process. However, a large portion of these proteins are of unknown function. This indicates that essential, yet undiscovered processes are conserved between $U$. maydis and humans.

\section{Results and Discussion}

In a first step we compared general sequence characteristics in coding regions of $U$. maydis, S. cerevisiae and H. sapiens using the fungal MIPS GenRE databases [32] and human data from the Ensembl database [33]. This included standard parameters, such as the average gene density or gene size (Table 1). In most of the analyzed parameters, including exon size, percent of coding region and average gene density, both fungi are closely related. Next, we did pair-wise comparisons of the whole proteome of $U$. maydis against $S$. cerevisiae and H. sapiens. For that a Java client stand-alone application was developed that was used to access the SIMAP retrieval layer (see Material and Methods; [30]). In this analysis, similarity in pair-wise comparison of proteins was indicated by the "evalue" or "\% identity over the length of the protein" and the median of these analyses was calculated. Surprisingly, we found that both median values were higher in the $U$. maydis-to-H. sapiens set compared to the U. maydis-to-S. cerevisiae analysis (Figure 1A). In contrast, when the proteome of $S$. cerevisiae was compared with $U$. maydis and $H$. sapiens, the expected outcome was that both fungi are more closely related than the budding yeast to humans (Figure 1B). These results added further support to our ini-

Table I: General characteristics of the coding region

\begin{tabular}{llccc}
\hline & & S. cerevisiae & U. maydis & H. sapiens \\
\hline Percent coding & $(\%)$ & 73.09 & 60.97 & $1.22-2.33$ \\
GC content & $(\%)$ & 39.58 & 56.26 & 52.10 \\
Average gene size & $(\mathrm{bP})$ & 1451 & 1747 & 1526 \\
Average gene density & $(\mathrm{kb} / \mathrm{gene})$ & 1.99 & 2.86 & 125.43 \\
Protein-coding genes & & 6127 & 6867 & $22762 \pi$ \\
Exons & $(\mathrm{bp})$ & 6482 & 9932 & 267941 \\
Average exon size & & 1372 & 1208 & 297 \\
Exons per gene & $(\%)$ & 1.058 & 2.45 & 6.149 \\
Genes with introns & & 4.81 & 27.73 & 97.96 \\
\hline
\end{tabular}

I the predicted number increases to 43576 when including splice variants. 

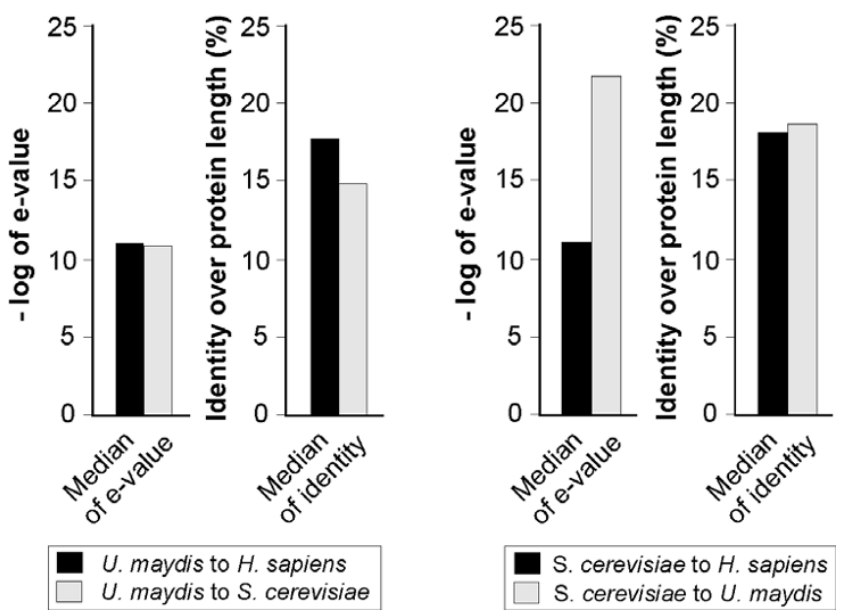

Figure I

Global comparison of the predicted proteome sequence of the fungi $U$. maydis, $S$. cerevisiae and $H$. sapiens. Based on the Smith-Waterman comparison of all proteins from genome A against all proteins of genomes $B$ the median e-value and the median \%-identity value were calculated. Note that $U$. maydis is more similar to humans than to its fungal cousin, whereas the proteome of $S$. cerevisiae is more closely to that of the corn smut.

tial assumption that $U$. maydis contains additional proteins that are highly conserved to humans, but that are absent from the fungal cousin S. cerevisiae.

Its general assumed that sequence conservation in proteins is a consequence of a similar function. However, even unrelated sequences show a certain degree of similarity, which increases in structural motifs such as coiled-coil domains that are found in $\sim 20 \%$ of all proteins in S. cerevisiae [34]. In order to determine the level of random sequence similarity, we compared unrelated proteins, including TBP (um10143), Myosin I (um11115) and FER1 (um00105) of $U$. maydis against each other. This analysis indicated that random sequence conservation reaches 1-5 \% identity. Next, we selected functionally unrelated proteins that contain coiled-coil regions (Table 2). A comparison of these proteins against each other revealed up to $16.9 \%$ sequence identity in those proteins that contained extended coiled-coil stretches (Yup1, Tpm2, Kin1 and Clip1; Table 2). Consequently, we considered only protein identity that was $20 \%$ or higher as indication for functional conservation, a value that corresponds with previous reports that conserved protein function is indicated by at least $18-20 \%$ protein sequence identity [35]. Following this criterion we redid our analysis and found that around 1738 proteins are shared by all three organisms (Figure 2), which represents $25-28 \%$ of the proteome of both fungi. Surprisingly, both fungi share 541 proteins, suggesting that only a minor portion (8$9 \%$ ) of the proteome is fungal specific. In contrast, $U$. maydis has 777 proteins that are more conserved in humans than in budding yeast, whereas $S$. cerevisiae shares only 514 proteins with $H$. sapiens. This again indicates that $U$. maydis is more closely related to humans than to its fungal cousin. However, a strict cut off at $20 \%$ could be misleading, as slight differences in sequence conservation around this border will not be recognized. In other words, a protein that has $20.1 \%$ identity between both fungi and $19.8 \%$ identity in humans would be considered as fungal specific. Moreover, a protein that shares $80 \%$ identity between both fungi, but only $20.1 \%$ with human will also not be identified. In order to cope with these problems, we included a more stringent criterion for our analysis and considered only those proteins that share $\geq 20 \%$ identity in two organisms, but found at $\geq 10 \%$ identity in the third partner. In this approach $U$. maydis still shares 587 fungal specific proteins with $S$. cerevisiae, but it has an even larger set of 651 proteins in common with $H$. sapiens (Figure 3). On the other hand, only 287 proteins of S. cerevisiae had a conserved counterpart in the human genome (not shown).

The results presented so far were based on a $20 \%$ identity over the total protein length. However, many proteins perform specific functions at short domains and functional orthologues might be overlooked when comparing whole proteins. A striking example is the microtubule plus-end binding protein Clip1 in U. maydis. It was shown that this protein binds to microtubule plus-ends and contains a CAP-Glu domain and two zinc finger

Table 2: Analysis of coiled-coil domain containing representatives from $U$. maydis

\begin{tabular}{|c|c|c|c|c|}
\hline MIPS ID & Gene & Function & Position & $\%$ identity \\
\hline um0379I & Ums2 & Heat shock $70 \mathrm{kd}$ protein & $507-539$ & $3.8-7.4$ \\
\hline um05406 & Yupl & t-SNARE & $249-286$ & $3.2-14.1$ \\
\hline um I I985 & Tpm2 & Tropomyosin & $|-76,7-16|$ & $3.1-13$ \\
\hline um04372 & Dyn2 & Dynein heavy chain & $89-156,311-387,663-699$ & $3.6-8.5$ \\
\hline um04555 & Myo5 & Myosin V & $958-1014,1022-1078,1079-1116$ & $3.5-11.3$ \\
\hline um06338 & ClipI & Microtubule binding protein & $56 \mathrm{I}-607,634-660,66 \mathrm{I}-708,709-76 \mathrm{I}, 822-867,868-94 \mathrm{I}, \mid 104 \mathrm{I}-1082$ & $10.0-16.9$ \\
\hline um04218 & Kin I & Motor protein & $346-378,44 I-55 I, 604-675,688-736,807-873,874-905$ & $9.4-14.3$ \\
\hline
\end{tabular}

I \% identity over entire protein length to unrelated coiled-coil proteins 
domains [22]. All these features are also typical for the human orthologue CLIP170 [36,37]. However, the overall sequence identity between Clip1 and CLIP170 is only at $19.5 \%$, and significant sequence conservation is only found in the CAP-Glu domain (aa 221-266; prosite motif analysed with ProfileScan; [38]; 50\% identity). Therefore, we considered it likely that our analysis has not covered all functional orthologues between the three organisms. Thus, we extended our analysis and compared sequences at a lower limit of 50 aa sequence overlap, which would cover short domains such as the FHA-domain in kinesin3 (57 aa, PFAM) or the mentioned CAP-Glu domain in Clip1 (65 aa, PFAM) and applied the same criterion as before ( $\geq 20 \%$ identity between two organisms in the overlapping region and $10 \%$ less in the third partner). This approach identified an additional 30 proteins being conserved between U. maydis and humans (now including Clip1), and 35 in case of a comparison of U. maydis and $\mathrm{S}$. cerevisiae. The number of proteins conserved between humans and budding yeast increased by 25 additional proteins (Figure 4).

Taken both analyses together our bioinformatic approach revealed 681 homologues between $U$. maydis and humans. In order to better define the orthology relationship among these genes we next performed a best bidirectional hit analysis assuming that orthologous proteins would identify the partner when searching in both directions. Making use of the SIMAP database we found that 620 proteins ( $~ 91 \%$ of $U$. maydis-to-H. sapiens set) fulfil this criterion (see Additional file 1). Thus, our data suggest that $\sim 10 \%$ of the $U$. maydis proteins have a role in cellular processes that are most likely conserved in the human cell.

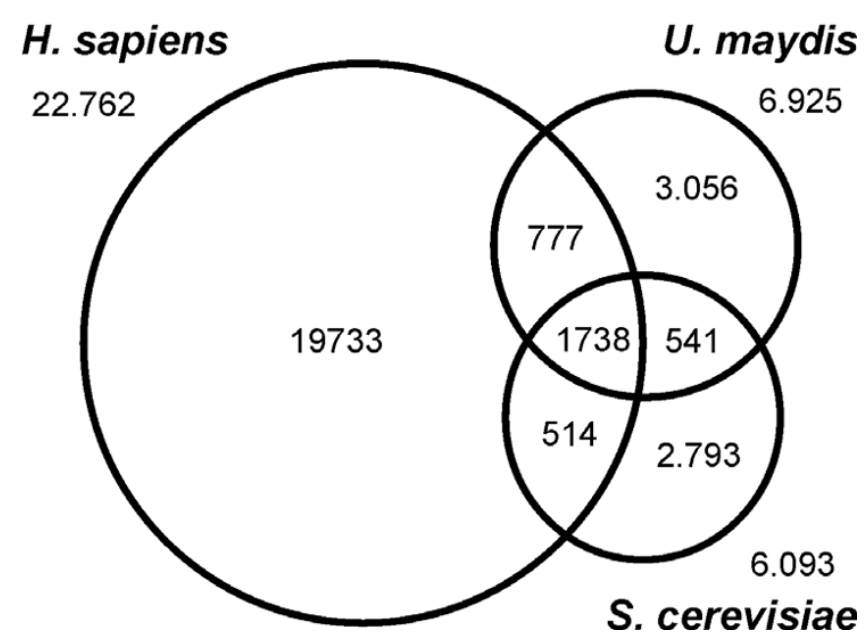

Figure 2

Comparison of the proteome of U. maydis, S. cerevisiae and $H$. sapiens using cut-off criteria of $20 \%$ identity over the total protein length.
On the other hand, budding yeast and humans share 312 unique proteins and yeast and $U$. maydis have 622 proteins in common (Figure 4). The most obvious next question therefore was whether theses proteins can be grouped in functional clusters. In order to address this question, we made use of the Functional Catalogue DataBase (FunCatDB; [39]) that summarizes functional annotation for proteomes from different organisms by assigning proteins of interest to certain cellular processes. In addition, our method provides a p-value that correlates the presence of proteins in the set to the expected abundance of proteins in the same functional groups in the proteome. We first analyzed proteins that are exclusively shared by both fungi and focused on those proteins, which are overrepresented in certain functional categories, whereas proteins that have a functional annotation but are not enriched in functional classes are not listed (Table 3A). According to our expectation, U. maydis and S. cerevisiae have proteins in common that are essential for their uni-cellular life style. This includes the detoxification machineries and proteins that are involved in spore formation (Table 3; numbers represent the number of proteins in the cellular process listed in the table). In addition, both organisms contain 96 proteins that do not fall in any functional category and are therefore classified as "unknown function" The second set consists of proteins found in humans and $U$. maydis and also represents numerous cellular processes, including amino acid degradation, oxidation of fatty acids, mRNA splicing and modification, protein modification and degradation and G-protein mediated signalling (Table 3B). However, about one third of all proteins in this set (222; Table 3) are neither found in the FunCat nor in Clusters of eukaryotic orthologous groups in the COGs database [40]; [41], indicating that their function is not yet known ("unknown function"). Finally, we analyzed the S. cerevisiae-to-H. sapiens homology set and again found some cellular processes, including tRNA modification, secondary biosynthesis and protein fate and modification that are overrepresented in this protein set (Table 3C). In contrast to the previous comparison, only 46 of the 312 proteins that are unique for yeast and human were without functional prediction in FunCat or the COGs database.

Our analysis revealed that $U$. maydis and human share numerous proteins, and analysis of these might give insights into the molecular basis of human diseases. A good example for such a role for $U$. maydis is the analysis of the breast cancer susceptibility gene BRCA2 that confers a high risk of breast cancer and is the focus of cancer research since its discovery in the mid 1990s $[42,43]$. The activity of BRCA2 is not well-understood, which might in part be due to the fact that it was not found in the model system S. cerevisiae. Recently a BRCA2 homologue (Brh2; um03200) was identified in a screen for DNA-repair 

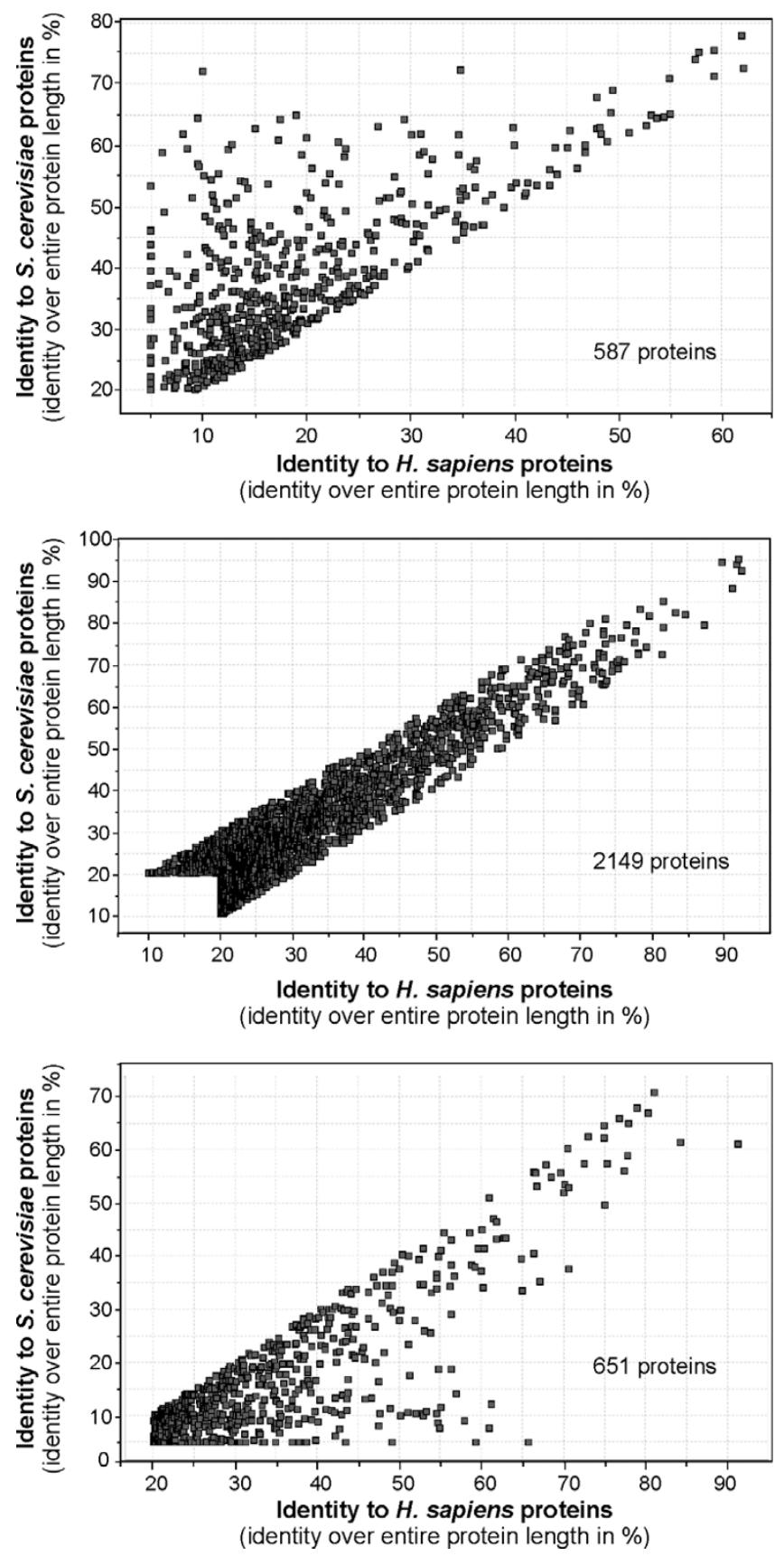

Figure 3

Genome-wide protein homology correlation of $U$. maydis against humans and S. cerevisiae using the \% identity over entire protein length. Depicted are predicted $U$. maydis proteins that show $>20 \%$ identity to one partner and $>10 \%$ less identity to the other. Most proteins (2I59) are conserved at similar levels in S. cerevisiae and H. sapiens (B). However, 587 proteins are more closely related to $S$. cerevisiae $(A)$, whereas an even larger number of $65 \mathrm{I}$ are more similar to humans (C). Note that this analysis show "\% identity over the total protein length" and thus does not include proteins that share sequence similarity only within a domain.

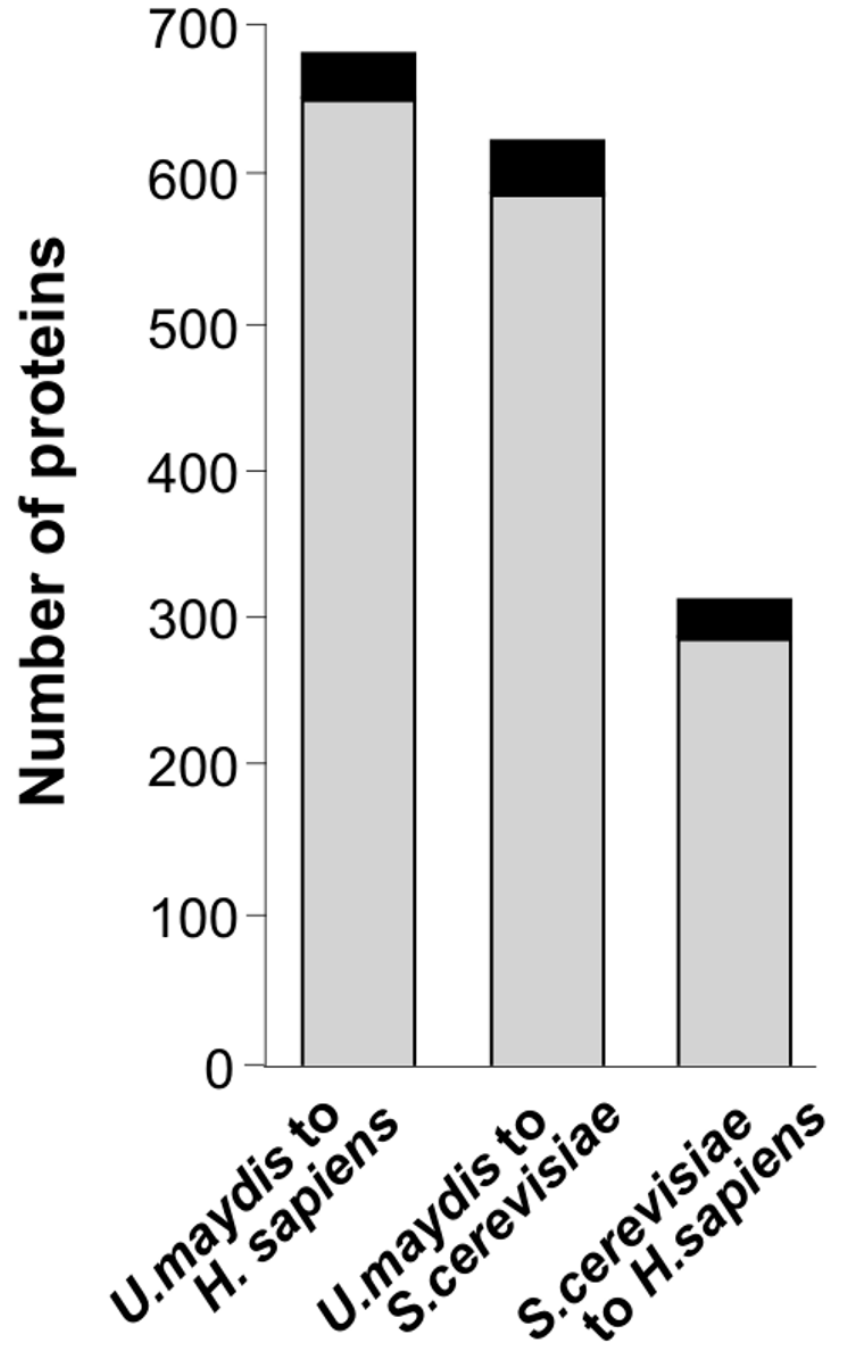

\section{Conservation only in domains Conservation over entire protein}

\section{Figure 4}

Total number of predicted proteins that show significant similarity ( $>20 \%$ identity to one partner and > $10 \%$ less identity to the other) over their entire length (grey bar) or within domains of at least 50 amino acids (black bar).

defective mutants [44], and it was shown that Brh2 enables recombinational DNA repair by controlling Rad51 $[45,46]$. Indeed, the $U$. maydis Rad51 is one of seven proteins that are implied in DNA repair and that are part of the 681 proteins identified to be highly conserved in humans and $U$. maydis. In order to gain insights into a role of $U$. maydis genes in human diseases we used the Genetic Association Database [47], which is an archive of all published knowledge of molecular disorders in humans. After 
Table 3: Functional classification of predicted proteins

\begin{tabular}{|c|c|c|}
\hline & Protein number $\pi$ & P-value \\
\hline \multicolumn{3}{|l|}{ A. U. maydis - S. cerevisiae* } \\
\hline I. Biosynthesis* & 75 & $1,7 e-33$ \\
\hline 2. Spore formation/budding/cell polarity & 68 & $1.9 \mathrm{e}-15$ \\
\hline 3. Transport** & 49 & $1.3 e-18$ \\
\hline 4. Ion homeostasis & 36 & $4.2 \mathrm{e}-12$ \\
\hline 5. Stress response $\$$ & 35 & $7.5 \mathrm{e}-11$ \\
\hline 6. Detoxification/Resistance & 29 & $1.4 \mathrm{e}-10$ \\
\hline 7. Unknown function ${ }^{\dagger}$ & 96 & I \\
\hline \multicolumn{3}{|l|}{ B. U. maydis - H. sapiens* } \\
\hline I. mRNA splicing & 38 & $6.9 e-17$ \\
\hline 2. Protein modification/degradation & 29 & $2.4 \mathrm{e}-04$ \\
\hline 3. G-protein-mediated signalling & 17 & $3,6 \mathrm{e}-06$ \\
\hline 4. Amino acid degradation & 14 & $4.4 \mathrm{e}-09$ \\
\hline 5. Oxidation of fatty acids & 10 & I.le-05 \\
\hline 6. Unknown functiont & 222 & I \\
\hline \multicolumn{3}{|l|}{ C. S. cerevisiae - H. sapiens* } \\
\hline I. Biosynthesis, secondary§ & 105 & $3.0 e-05$ \\
\hline 2. Protein fate, - modification & 89 & $7.4 \mathrm{e}-06$ \\
\hline 3. Oxidative stress response & 8 & $3.7 e-03$ \\
\hline 4. tRNA modification & 7 & $3.4 e-03$ \\
\hline 5. Unknown functiont & 46 & 1 \\
\hline
\end{tabular}

*Note that proteins can appear in more than one functional category. In addition, numerous proteins are assigned to additional functional classes that are not listed.

T Only those predicted proteins were considered that (I) show more than $20 \%$ identity with either in both organisms and (2) show at least $10 \%$ less homology in the third partner

*Amino acids/vitamins/cofactors

**Amino acids/lipids/carbohydrates

\$Salt and oxidative stress

\$Polyamines, vitamins, co-factors and other secondary products

tPredicted proteins are not found in the COGs or FunCat database

adjusting the nomenclature (see Material and Methods) all proteins that matched were analyzed and then classified according to main disease classes. This analysis revealed that 42 proteins of the $U$. maydis-to-H. sapiens set are implicated in diseases, including cancer (8 proteins), cardiovascular disorders (7 proteins) and defects in metabolism (11). In addition, 22 proteins (Figure 5, "Others") were found that are implicated in various defined diseases or whose roles are not yet understood (Figure 5). Among these proteins are prominent oncogenes such as ERCC1 (um06219) and ERCC4 (um10396), which participate in excision repair of DNA, and when mutated cause various types of cancer, including non-small-cell lung cancer [48]. Another example is the KRAS protein (um01643), the GTP/GTP-binding protein acting in intracellular signal transduction (overview in [49]) that also is involved in cancer formation. Most interesting, we found several disease-related proteins that most likely are not present in the model system $S$. cerevisiae ( $\leq 14 \%$ identity; Table 4$)$. This group includes ACADM (um01049) and ACADS (um01466), which encode acyl-CoA dehydrogenases (Table 4). Mutants in these genes cause medium-chain-acyl-CoA dehydroge- nase deficiency [50] and short-chain-acyl-CoA dehydrogenase deficiency in humans [51].

Finally, it is important to note that the yeast $S$. cerevisiae also shares 13 disease-related proteins with humans that are significantly less conserved in $U$. maydis (Figure 5), and 4 of these proteins are not present in $U$. maydis $(<$ $10 \%)$. Interestingly, a third of all disease-related proteins in yeast are involved in diabetes. Among these is ADIPOR2 (YOL002c), an adiponectin receptor, which when deleted in mice promotes type 2 diabetes [52] and PYGL (YPR160c), which when mutated causes Glycogen phosphorylase deficiency, resulting in diabetes mellitus type 1 in humans [53].

\section{Conclusion}

Fungal model systems, such as $S$. cerevisiae have greatly enhanced our knowledge of basic cell biology, which is in part due to numerous technical advantages and the published genome. However, some cellular processes that are important in humans are highly modified or are not even present in this fungus. Examples are microtubule-based transport that is essential in elongated neurons, but virtu- 
Table 4: Disease-related proteins with counterparts in U. maydis

\begin{tabular}{|c|c|c|c|c|c|c|}
\hline Protein & Function & MIPS ID & Diseases & U. maydis* & S. cerevisiae* & Ref.** \\
\hline ACADM & acyl-CoA dehydrogenase & Um01049 & acyl-CoA dehydrogenase deficiency & 58.0 & 11.6 & [68] \\
\hline ACADS & acyl-CoA dehydrogenase & Um04833 & acyl-CoA dehydrogenase deficiency & 35.2 & 11.2 & {$[69]$} \\
\hline BZRP/PBR & Benzodiazepine receptor & Um06406 & Cancer & 27.4 & 10.1 & [70] \\
\hline CESI & Carboxylesterase & Um0607I & Cancer & 27.6 & 9.3 & [7I] \\
\hline CLPTMI & unknown & Um02586 & Cleft lip development & 30.5 & II.I & [72] \\
\hline DDC & Dopa decarboxylase & Um06083 & Parkinsons & 34.7 & 11.0 & {$[73]$} \\
\hline DOCK3 & Dedicator of cytokinesis 3 & Um0II78 & Attention deficit hyperactivity disorder & 23.1 & 12.3 & [74] \\
\hline EPHXI & Epoxide hydrolase & Um01938 & Cancer & 36.6 & 10.1 & [74] \\
\hline $\mathrm{GCDH}$ & glutaryl-CoA dehydrogenase & Um01335 & glutaryl-CoA dehydrogenase deficiency & 54.1 & 13.9 & {$[75]$} \\
\hline HEXA & Hexosaminidase A & Um00695 & Tay-Sachs disease & 20.1 & 12.7 & {$[76]$} \\
\hline HMGCL & Methylglutaryl CoA lyase & Um0200I & methylglutaric CoA lyase deficiency & 43.6 & 11.4 & {$[77]$} \\
\hline PIMT/PCMTI & Methyl-transferase & Uml I483 & Type I diabetes & 48.9 & 9.3 & {$[78]$} \\
\hline PRSSI 6 & serine protease & Uml009I & $\begin{array}{l}\text { Diabetes mellitus/susceptibility to } \\
\text { autoimmunity }\end{array}$ & 20.7 & 6.6 & [79] \\
\hline UGCG & Glucosyl-ceramide synthase & Um04496 & induced apoptosis & 22.3 & 8.1 & {$[80]$} \\
\hline
\end{tabular}

IIdentification tag for search at the MIPS server [13]

* Values are given as percent identity to human protein, all the $U$. maydis hits are best bidirectional

** Reference for the human protein/disease

ally absent from S. cerevisiae. Consequently, some proteins, such as the motor proteins kinesin- 1 and kinesin-3 are not encoded by the genome of the budding yeast. Experimental evidence from work in $U$. maydis indicated that this fungus could fill in this gap. This organism has a long history as a model system for DNA repair and recombination, and shows additional similarities to human cells, such as long-distance transport and an open mitosis. Consequently, proteins like kinesin- 1 and kinesin- 3 are present and are highly conserved in $U$. maydis. Indeed, as

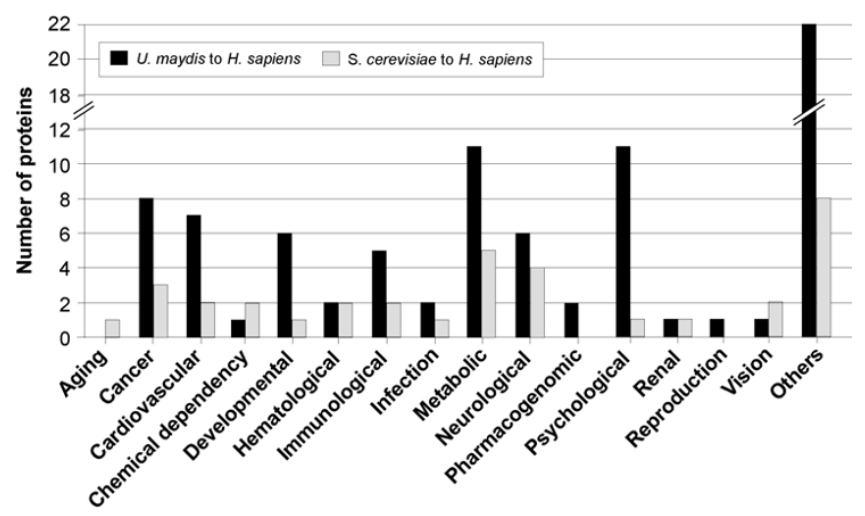

Figure 5

Proteins of the $U$. maydis $-H$. sapiens and $S$. cerevisiae $-H$. sapiens homology sets, which human counterpart have a predicted role in diseases. The classification was done according to main disease class in the genetic association database [47]. Note that $U$. maydis contains numerous genes that are thought to be involved in psychological disorders, such as ERCCI and ERCC4. much as $\sim 10 \%$ of all $U$. maydis proteins have highly conserved counterparts in humans, but are not found or are significant less conserved in yeast. On the first glimpse this finding is surprising. However, genomic data indicate that fungi are an extremely divers taxon that covers around one billion years of divergent evolution [54]. The unexpectedly high conservation between $U$. maydis and humans might suggest that both organisms share some conserved cellular processes. However, it is important to note that functional predictions based on sequence homology can just be a first step towards an understanding of the cellular function. Careful experimental work is needed to further prove that $U$. maydis helps understanding the molecular basis of human diseases.

\section{Methods}

\section{General Hardware and software}

All software was compiled and run on one or more workstations with Fedora Linux or Alpha processor and Tru64 (formerly known as DEC-Unix) with compiled software for OSF v5.1. Unless otherwise noted, data mining and analyses was carried out using the Protein Mapping and comparison Tool PROMPT [55] an java application including the statistic package $\mathrm{R}$, scripts written in Perl [56] or using own Java applications [57] developed with the open source platform Eclipse [58], each of which are available on request. In all cases, data is stored in mySQL $4 / 5$ [59] or Oracle 9i [60] databases referred to as the MIPS Fungal Genome Databases (U. maydis, S. cerevisiae) and the Functional Catalogue Database (FunCatDB) [39]. 


\section{Homology analysis}

In order to determine the homology set of the proteomes of $U$. maydis, S. cerevisiae and H. sapiens we used the SIMAP [61], an exhaustive application containing all significant pre-calculated similarity scores of the Smith-Waterman alignment algorithm [62] of protein pairs. The database contains more than 30 million proteins, including the current versions of the $S$. cerevisiae and $U$. maydis proteomes and all available human protein sequences and is well suited to speed up the search for biological meaningful hits [30]. In order to access this database, a java application was generated using the eclipse framework (version 3.2.1) for communication with the SIMAP retrieval layer (due to the huge size of the binary hit file $700 \mathrm{G}$ and ongoing internal changes, a direct access is not permitted). This allowed for the access of further stored information (sequence ID, Smith-Waterman score, Identity score, Similarity score, overlap size of the pair-wise alignment and Start and Stop coordinates of the alignment in both proteins), as well as sorting and filtering to specific criteria.

Additionally, we implemented in our client application the "identity over length" sorting procedure to tackle the length dependency, an important task for transferring functional attributes. With help of this application and specifications, represented in single task files, a genome wide retrieving, sorting and taxonomic as well as homology filtering was feasible. Homologous proteins were identified using the whole-genome protein sets of U. maydis (MUMDB), e.g. S. cerevisiae (CYGD) and H. sapiens (UniProtKB Refseq). For various cut-offs of homology assignments for proteins and domains see also the specific result parts. Further we integrated a SIMAP access for data visualization of various taxonomy spaces (e.g. human) in each of our organism specific databases in the Genome Research Environment [63].

In contrast to our so far used "\% identity over length" criteria we sorted the hits in the domain search by the standard e-value and considered the \% identity in the overlap at a cut-off length of 50 aa.

\section{Sequence similarity background of structural (coiled-coil) domains}

In order to predict coiled-coil domains we used Paircoil2 $[64,65])$. This tool uses pair-wise residue probabilities to detect coiled-coil motifs in protein sequence data and achieves $98 \%$ sensitivity and $97 \%$ specificity on known coiled coils in leave-family-out cross-validation. We took from each of the coiled-coil families (dynein, heat-shock factor, intermediate filament-like, kinesin-like, myosin, snare and tropomyosin) one representative $U$. maydis protein and detected the domain position (Table 2). Next we calculated the \% identity over the entire protein for the 7 examples to each other and additionally to the $U$. maydis proteome. We filtered out the known related protein and thus we expected that like in yeast the coilome is around $20 \%$ of the proteome [34]. Then we estimated the random \% identity, which is by far lower than the range of the $30^{\text {th }}$ hit until the best unrelated hit shown in Table 2. Please note that $19.0 \%$ - 29.1\% identity in the overlap region can be found for unrelated coiled-coil domain containing proteins.

\section{Functional data and analysis}

The systematic classification of protein function is of great importance in functional genomics, as it organizes our thinking about the biological roles of proteins. The MIPS Functional Catalogue Database resource (FunCatDB; [39]) contains functional proteome information (collected literature as well as homology transformations) across organisms based on the hierarchical classification scheme FunCat [31]. Included in this database are high value manual functional annotation for the S. cerevisiae proteome as well as homology assignments to already manually annotated proteins, such as a quality value of $>$ $25 \%$ identity over length [35] for the U. maydis proteome. For each set of proteins we obtained and analyzed their functional distribution and the statistical significance of functional similarity groups, based on the p- value calculation of each group from the set against the corresponding whole genome reference set. To not miss a function due to possible incomplete annotation, we calculated with help of our automated Pedant analysis system $[66,67]$ for all the $U$. maydis proteins the similarity to the Clusters of eukaryotic groups (KOGs; [40,41] using a cutoff of $20 \%$ identity. The eukaryotic orthologous groups (KOGs) include proteins from 7 eukaryotic genomes including $H$. sapiens and S. cerevisiae. The current KOG set consists of 4852 clusters of orthologous proteins, which are annotated to a set of distinct main functions.

\section{Diseases and disorder analysis}

In order to study the connection of homologous proteins to human diseases and disorders, we first mapped all human codes from SwissProt, ensemble, NCBI and Unicode to the official human gene nomenclature. We then applied the batch tool at the Genetic Association Database, an archive of human genetic association studies of complex diseases and disorders, for analysis of genetic association [47] and their classification in main disease classes. Additionally, we performed a literature search for all identified homologous human proteins to obtain the current and precise cellular functions as well as diseases.

\section{Authors' contributions}

MM carried out the conception and design of the study and implemented the computational studies and statistical analysis. Further MM participated in the interpretation of data and the manuscript preparation. GS conceived of 
the study and participated in the interpretation of data and the manuscript preparation. All authors read and approved the final manuscript.

\section{Additional material}

\section{Additional file 1}

List of all Ustilago maydis proteins with higher conservation to Homo sapiens than to Saccharomyces cerevisiae. Listed are the $\mathrm{U}$ maydis code, the \%identity over protein length to $\mathrm{H}$. sapiens and the \% identity over protein length to $\mathrm{S}$. cerevisiae, based on the Smith-Waterman comparison of all proteins between the genomes, a flag (1) for a best bidirectional hit, $\mathrm{H}$. sapiens code, the alternative best reciprocal $\mathrm{U}$. maydis code, the \%identity over protein length of the best reciprocal hit and comments (The U. maydis annotation is still ongoing, please find here existing new gene models). List of alternative Best Recipropal Hits of the $\mathrm{U}$. maydis-to-H. sapiens set. Listed are all best recipropal $\mathrm{U}$. maydis codes, their amount and their relation to the $\mathrm{U}$. maydis-to-H. sapiens set.

Click here for file

[http://www.biomedcentral.com/content/supplementary/1471-

2164-8-473-S1.pdf]

\section{Acknowledgements}

We thank Roland Arnold for help developing a Java client stand-alone application for communication with the SIMAP retrieval layer and Gertrud Mannhaupt and Ullrich Güldener for critical reading of the manuscript.

\section{References}

I. Evans CS, Hedger JN: Degradation of plant cell wall polymers. In Fungi in bioremediation Edited by: Gadd GM. Cambridge, United kingdom: Cambridge University Press; 200I:I-26.

2. Smith SE, Read DJ: Mycorrhizal symbiosis. New York: Academic Press, Inc; 1997.

3. Tournas VH: Spoilage of vegetable crops by bacteria and fungi and related health hazards. Crit Rev Microbiol 2005, 31 (I):33-44.

4. San-Blas G, Travassos LR, Fries BC, Goldman DL, Casadevall A, Carmona AK, Barros TF, Puccia R, Hostetter MK, Shanks SG, et al.: Fungal morphogenesis and virulence. Med Mycol 2000, 38(Suppl I):79-86.

5. Baldauf SL, Palmer JD: Animals and fungi are each other's closest relatives: congruent evidence from multiple proteins. Proc Natl Acad Sci U S A 1993, 90(24): I I 558- I 562.

6. Wainright PO, Hinkle G, Sogin ML, Stickel SK: Monophyletic origins of the metazoa: an evolutionary link with fungi. Science 1993, 260(5 I06):340-342.

7. Goffeau A, Barrell BG, Bussey H, Davis RW, Dujon B, Feldmann H, Galibert F, Hoheisel JD, Jacq C, Johnston M, et al.: Life with $\mathbf{6 0 0 0}$ genes. Science 1996, 274(5287):546, 563-547.

8. Comprehensive Yeast Genome Database (CYGD) [http:// mips.gsf.de/genre/proj/yeast/]

9. Ris H: Primitive mitotic mechanisms. Biosystems 1975, 7(34):298-30I.

10. Pruyne D, Legesse-Miller A, Gao L, Dong Y, Bretscher A: Mechanisms of polarized growth and organelle segregation in yeast. Annu Rev Cell Dev Biol 2004, 20:559-59I.

II. Xu JR, Peng YL, Dickman MB, Sharon A: The dawn of fungal pathogen genomics. Annu Rev Phytopathol 2006, 44:337-366.

12. Kamper J, Kahmann R, Bolker M, Ma LJ, Brefort T, Saville BJ, Banuett F, Kronstad JW, Gold SE, Muller O, et al: Insights from the genome of the biotrophic fungal plant pathogen Ustilago maydis. Nature 2006, 444(71 I5):97-I0I.

13. MIPS Ustilago maydis database MUMDB [http://mips.gsf.de/ genre/proj/ustilago/]
14. Mewes HW, Amid C, Arnold R, Frishman D, Guldener U, Mannhaupt G, Munsterkotter M, Pagel P, Strack N, Stumpflen V, et al.: MIPS: analysis and annotation of proteins from whole genomes. Nucleic Acids Res 2004:D4I-44.

15. Feldbrugge M, Kamper J, Steinberg G, Kahmann R: Regulation of mating and pathogenic development in Ustilago maydis. Curr Opin Microbiol 2004, 7(6):666-672.

16. Perez-Martin J, Castillo-Lluva S, Sgarlata C, Flor-Parra I, Mielnichuk N, Torreblanca J, Carbo N: Pathocycles: Ustilago maydis as a model to study the relationships between cell cycle and virulence in pathogenic fungi. Mol Genet Genomics 2006, 276(3):2II-229.

17. Klosterman SJ, Perlin MH, Garcia-Pedrajas M, Covert SF, Gold SE: Genetics of morphogenesis and pathogenic development of Ustilago maydis. Adv Genet 2007, 57: 1-47.

18. Holliday R: A mechanism for gene conversion in fungi. Genet Res 1964, 5:282-290.

19. Holliday R: Early studies on recombination and DNA repair in Ustilago maydis. DNA Repair (Amst) 2004, 3(6):67|-682.

20. Steinberg G: Tracks for traffic: microtubules in the plant pathogen Ustilago maydis. New Phytol 2007, 174(4):721-733.

21. Schuchardt I, Assmann D, Thines E, Schuberth C, Steinberg G: Myosin-V, Kinesin-I, and Kinesin-3 cooperate in hyphal growth of the fungus Ustilago maydis. Mol Biol Cell 2005, I6(II):5191-5201.

22. Lenz JH, Schuchardt I, Straube A, Steinberg G: A dynein loading zone for retrograde endosome motility at microtubule plusends. Embo / 2006, 25(I I):2275-2286.

23. Wedlich-Soldner R, Straube A, Friedrich MW, Steinberg G: A balance of KIF IA-like kinesin and dynein organizes early endosomes in the fungus Ustilago maydis. Embo J 2002, 2I(I 2):2946-2957.

24. Lippincott-Schwartz J: Cell biology: ripping up the nuclear envelope. Nature 2002, 4 I 6(6876):3|-32.

25. Beaudouin J, Gerlich D, Daigle N, Eils R, Ellenberg J: Nuclear envelope breakdown proceeds by microtubule-induced tearing of the lamina. Cell 2002, I08(I):83-96.

26. Straube A, Weber I, Steinberg G: A novel mechanism of nuclear envelope break-down in a fungus: nuclear migration strips off the envelope. Embo J 2005, 24(9):1674-I685.

27. Theisen U, Straube A, Steinberg G: Dynamic rearrangement of nucleoporins during fungal "open" mitosis. Mol Biol Cell 2007. in revision

28. Belgareh N, Rabut G, Bai SW, van Overbeek M, Beaudouin J, Daigle N, Zatsepina OV, Pasteau F, Labas V, Fromont-Racine M, et al:: An evolutionarily conserved NPC subcomplex, which redistributes in part to kinetochores in mammalian cells. J Cell Biol 200I, 154(6): I I47-II60.

29. Loiodice I, Alves A, Rabut G, Van Overbeek M, Ellenberg J, Sibarita JB, Doye V: The entire Nup/07-160 complex, including three new members, is targeted as one entity to kinetochores in mitosis. Mol Biol Cell 2004, 15(7):3333-3344.

30. Arnold R, Rattei T, Tischler P, Truong MD, Stumpflen V, Mewes W: SIMAP - The similarity matrix of proteins. Bioinformatics 2005 , 2 I(Suppl 2):ii42-ii46.

31. Ruepp A, Zollner A, Maier D, Albermann K, Hani J, Mokrejs M, Tetko I, Guldener U, Mannhaupt G, Munsterkotter M, et al:: The FunCat, a functional annotation scheme for systematic classification of proteins from whole genomes. Nucleic Acids Res 2004, 32(18):5539-5545.

32. The fungal MIPS GenRE databases [http://mips.gsf.de/projects/ fungi/]

33. Ensembl [http://www.ensembl.org]

34. Barbara KE, Willis KA, Haley TM, Deminoff SJ, Santangelo GM: Coiled coil structures and transcription: an analysis of the $S$. cerevisiae coilome. Mol Genet Genomics 2007, 278(2): 135-147.

35. Wilson CA, Kreychman J, Gerstein M: Assessing annotation transfer for genomics: quantifying the relations between protein sequence, structure and function through traditional and probabilistic scores. J Mol Biol 2000, 297(I):233-249.

36. Griparic L, Volosky JM, Keller TC 3rd: Cloning and expression of chicken CLIP-I70 and restin isoforms. Gene 1998, 206(2): 195-208.

37. Diamantopoulos GS, Perez F, Goodson HV, Batelier G, Melki R, Kreis TE, Rickard JE: Dynamic localization of CLIP-I 70 to microtu- 
bule plus ends is coupled to microtubule assembly. / Cell Biol 1999, 144(1):99-1 I2.

38. ProfileScan [http://www.expasy.ch/prosite]

39. Functional Catalogue Database (FunCatDB) [http:// mips.gsf.de/proj/funcatDB/]

40. Clusters of orthologous groups (COGs) database [http:// www.ncbi.nlm.nih.gov/COG/]

41. Tatusov RL, Fedorova ND, Jackson JD, Jacobs AR, Kiryutin B, Koonin EV, Krylov DM, Mazumder R, Mekhedov SL, Nikolskaya AN, et al.: The COG database: an updated version includes eukaryotes. BMC Bioinformatics 2003, 4:41.

42. Bishop DT: BRCA1, BRCA2, BRCA3 ... a myriad of breast cancer genes. Eur J Cancer 1994, 30A(I 2): 1738-1739.

43. Narod SA, Foulkes WD: BRCAI and BRCA2: 1994 and beyond. Nat Rev Cancer 2004, 4(9):665-676.

44. Kojic M, Kostrub CF, Buchman AR, Holloman WK: BRCA2 homolog required for proficiency in DNA repair, recombination, and genome stability in Ustilago maydis. Mol Cell 2002, 10(3):683-691.

45. Kojic M, Zhou Q, Lisby M, Holloman WK: Rec2 interplay with both Brh2 and Rad5I balances recombinational repair in Ustilago maydis. Mol Cell Biol 2006, 26(2):678-688.

46. Zhou Q, Kojic M, Cao Z, Lisby M, Mazloum NA, Holloman WK: Dss I interaction with Brh2 as a regulatory mechanism for recombinational repair. Mol Cell Biol 2007, 27(7):25 I2-2526.

47. Genetic Association Database [http://geneticassocia tiondb.nih.gov/]

48. Kiyohara C, Yoshimasu K: Genetic polymorphisms in the nucleotide excision repair pathway and lung cancer risk: a meta-analysis. Int J Med Sci 2007, 4(2):59-7I.

49. Goddard NC, Mclntyre A, Summersgill B, Gilbert D, Kitazawa S, Shipley J: KIT and RAS signalling pathways in testicular germ cell tumours: new data and a review of the literature. Int J Androl 2007.

50. Nennstiel-Ratzel U, Arenz S, Maier EM, Knerr I, Baumkotter J, Roschinger W, Liebl B, Hadorn HB, Roscher AA, von Kries R: Reduced incidence of severe metabolic crisis or death in children with medium chain acyl-CoA dehydrogenase deficiency homozygous for c.985A $>\mathbf{G}$ identified by neonatal screening. Mol Genet Metab 2005, 85(2): I57-I59.

51. van Maldegem BT, Waterham HR, Duran M, van der Vlies M, van Woerden CS, Bobu LL, Wanders RJ, Wijburg FA: The 625G>A SCAD gene variant is common but not associated with increased C4-carnitine in newborn blood spots. J Inherit Metab Dis 2005, 28(4):557-562.

52. Liu Y, Michael MD, Kash S, Bensch WR, Monia BP, Murray SF, Otto KA, Syed SK, Bhanot S, Sloop KW, et al.: Deficiency of adiponectin receptor 2 reduces diet-induced insulin resistance but promotes type 2 diabetes. Endocrinology 2007, 148:683-692.

53. Tomihira M, Kawasaki E, Nakajima H, Imamura Y, Sato Y, Sata M, Kage $M$, Sugie $H$, Nunoi $K$ : Intermittent and recurrent hepatomegaly due to glycogen storage in a patient with type I diabetes: genetic analysis of the liver glycogen phosphorylase gene (PYGL). Diabetes Res Clin Pract 2004, 65(2): I75-I82.

54. Hedges SB: The origin and evolution of model organisms. Nat Rev Genet 2002, 3(II):838-849.

55. Schmidt T, Frishman D: PROMPT: a protein mapping and comparison tool. BMC Bioinformatics 2006, 7:331.

56. Perl [http://www.perl.org/]

57. Java [http://www.java.org/]

58. Eclipse [http://www.eclipse.org/]

59. mySQL 4/5 [http://www.mysal.com/]

60. Oracle $9 \mathrm{i}$ [http://www.oracle.com/]

61. SIMAP (SImilarity MAtrix of protein Sequences) [http:// mips.gsf.de/simap]

62. Smith TF, Waterman MS: Identification of common molecular subsequences. J Mol Biol I98I, I47(I): 195-197.

63. Genome Research Environment (GenRE) [http://mips.gsf.de/ genre/proi/genre/]

64. Paircoil2 [http://groups.csail.mit.edu/cb/paircoil2/]

65. McDonnell AV, Jiang T, Keating AE, Berger B: Paircoil2: improved prediction of coiled coils from sequence. Bioinformatics 2006, 22(3):356-358.

66. Pedant [http://pedant.gsf.de]
67. Riley ML, Schmidt T, Artamonova II, Wagner C, Volz A, Heumann K, Mewes HW, Frishman D: PEDANT genome database: 10 years online. Nucleic Acids Res 2007:D354-357.

68. Zschocke J, Schulze A, Lindner M, Fiesel S, Olgemoller K, Hoffmann GF, Penzien J, Ruiter JP, Wanders RJ, Mayatepek E: Molecular and functional characterisation of mild MCAD deficiency. Hum Genet 200I, I 08(5):404-408.

69. Andresen BS, Christensen E, Corydon TJ, Bross P, Pilgaard B, Wanders RJ, Ruiter JP, Simonsen H, Winter V, Knudsen I, et al.: Isolated 2-methylbutyrylglycinuria caused by short/branched-chain acyl-CoA dehydrogenase deficiency: identification of a new enzyme defect, resolution of its molecular basis, and evidence for distinct acyl-CoA dehydrogenases in isoleucine and valine metabolism. Am J Hum Genet 2000, 67(5): 1095-I I 03.

70. Maaser K, Grabowski P, Oezdem Y, Krahn A, Heine B, Stein H, Buhr $H$, Zeitz M, Scherubl H: Up-regulation of the peripheral benzodiazepine receptor during human colorectal carcinogenesis and tumor spread. Clin Cancer Res 2005, I I(5): I75I-I756.

71. Pavillard V, Agostini C, Richard S, Charasson V, Montaudon D, Robert J: Determinants of the cytotoxicity of irinotecan in two human colorectal tumor cell lines. Cancer Chemother Pharmacol 2002, 49(4):329-335

72. Ichikawa E, Watanabe A, Nakano Y, Akita S, Hirano A, Kinoshita A Kondo S, Kishino T, Uchiyama T, Niikawa N, et al.: PAX9 and TGFB3 are linked to susceptibility to nonsyndromic cleft lip with or without cleft palate in the Japanese: populationbased and family-based candidate gene analyses. J Hum Genet 2006, 5 I (I):38-46.

73. Opacka-Juffry J, Brooks DJ: L-dihydroxyphenylalanine and its decarboxylase: new ideas on their neuroregulatory roles. Mov Disord 1995, I 0(3):24I-249.

74. de Silva MG, Elliott K, Dahl HH, Fitzpatrick E, Wilcox S, Delatycki M, Williamson R, Efron $D$, Lynch M, Forrest S: Disruption of a novel member of a sodium/hydrogen exchanger family and DOCK3 is associated with an attention deficit hyperactivity disorder-like phenotype. J Med Genet 2003, 40( I 0):733-740.

75. Zschocke J, Quak E, Guldberg P, Hoffmann GF: Mutation analysis in glutaric aciduria type I. J Med Genet 2000, 37(3): I77-I8I.

76. Akli S, Chomel JC, Lacorte JM, Bachner L, Kahn A, Poenaru L: Ten novel mutations in the HEXA gene in non-Jewish Tay-Sachs patients. Hum Mol Genet 1993, 2(I):6I-67.

77. Cardoso ML, Rodrigues MR, Leao E, Martins E, Diogo L, Rodrigues E, Garcia P, Rolland MO, Vilarinho L: The E37X is a common HMGCL mutation in Portuguese patients with 3-hydroxy-3methylglutaric CoA lyase deficiency. Mol Genet Metab 2004, 82(4):334-338

78. Wagner AM, Cloos P, Bergholdt R, Boissy P, Andersen TL, Henriksen DB, Christiansen C, Christgau S, Pociot F, Nerup J: Post-translational protein modifications in type I diabetes: a role for the repair enzyme protein-L: -isoaspartate (D: -aspartate) 0 methyltransferase? Diabetologia 2007, 50(3):676-68I.

79. Cheunsuk S, Sparks R, Noveroske JK, Hsu T, Justice MJ, Gershwin $M E$, Gruen JR, Bowlus CL: Expression, genomic structure and mapping of the thymus specific protease prss 16: a candidate gene for insulin dependent diabetes mellitus susceptibility. Autoimmun 2002, 18(4):31I-316.

80. Turzanski J, Grundy M, Shang S, Russell N, Pallis M: P-glycoprotein is implicated in the inhibition of ceramide-induced apoptosis in TF-I acute myeloid leukemia cells by modulation of the glucosylceramide synthase pathway. Exp Hematol 2005, 33(I):62-72. 